\title{
Determinação do saldo radiativo na llha do Bananal, TO, com imagens orbitais ${ }^{1}$
}

\author{
Ana C. V. L. Gusmão², Bernardo B. da Silva ${ }^{3}$, \\ Suzana M. G. L. Montenegro ${ }^{4} \&$ Josiclêda D. G alvíncio ${ }^{3}$
}

\begin{abstract}
RESU M 0
O sensoriamento remoto possibilita identificar alterações que ocorrem na superfície terrestre, resultantes de fenômenos naturais e/ou antrópicos, muitas das quais podem ser diagnosticadas a partir do monitoramento das trocas radiativas à superfície. N este sentido, o presente estudo objetiva determinar 0 saldo da radiação à superfície apenas com o emprego de imagens TM-Landsat 5, na Ilha do Bananal, TO. Foram selecionadas imagens de 2005 sem presença de nuvens e gerados mapas do saldo de radiação instantâneo e diário utilizando-se o algoritmo SEBAL. O s dados obtidos foram validados com medições realizadas no interior da área estudada. 0 saldo de radiação instantâneo médio situou-se entre 488 e $535 \mathrm{~W} \mathrm{~m}^{-2}$, enquanto a variação espacial do saldo de radiação diário - $\mathrm{Rn},{ }_{24 \mathrm{~h}}$ médio, foi de 132,7 a $145,3 \mathrm{~W} \mathrm{~m}^{-2}$. As áreas antropizadas apresentaram redução no saldo radiativo. 0 Erro M édio Relativo, M édio Absoluto e a Raiz do Erro Q uadrático M édio, associados ao $\mathrm{Rn}_{24 \text { apresentaram valores }}$ de $1,95 \%, 2,78 \mathrm{~W} \mathrm{~m}^{-2}$ e 3,36 W m $\mathrm{W}^{-2}$, respectivamente, evidenciando a importância da técnica empregada.
\end{abstract}

Palavras-chave: Landsat 5, albedo, temperatura da superfície, balanço radiativo

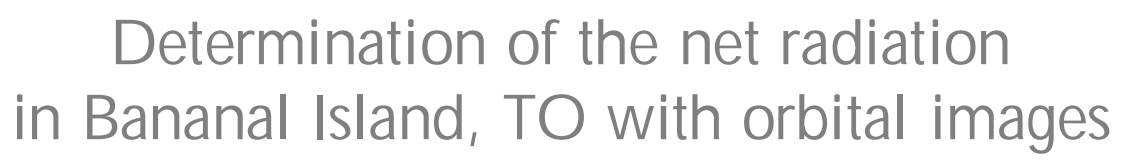

ABSTRACT

Remote sensing may be used to identify changes that occur in land use of Earth, resulting from natural and/or anthropic processes. Many of these changes can be diagnosed from the monitoring of radiative exchanges that take place at the surface. In this sense, this study aims to determine the net radiation at surface, by means of satellite images covering Bananal Island, in the State of Tocantins, Brazil. TM -Landsat 5 images obtained in 2005 (cloud free), were used to generate maps of instantaneous and daily net radiation $(\mathrm{Rn})$ based on SEBAL. The $\mathrm{Rn}$ values were validated with measurements performed in a micrometeorological tower installed within the Bananal Island. The instantaneous $R n$ values varied between 488.0 and $535.0 \mathrm{~W} \mathrm{~m}^{-2}$, while the daily $\mathrm{Rn}$ values varied between 132.7 and $145.3 \mathrm{~W} \mathrm{~m}^{-2}$. The disturbed areas showed decrease in net radiation. The daily net radiation presented relative mean error, mean absolute error and square root of mean square error equal to $1.95 \%, 2.78 \mathrm{~W} \mathrm{~m}^{-2}$ and $3.36 \mathrm{~W} \mathrm{~m}^{-2}$, respectively. According to the results, it may be concluded that the methodology used for $\mathrm{Rn}$ determination presented good accuracy.

Key words: Landsat 5, albedo, land surface temperature, radiation budget

\footnotetext{
${ }^{1}$ Parte da dissertação da primeira autora apresentada ao Programa de Pós-Graduação em M eteorologia

2 GRH/UFPE. Av. Acad. Hélio Ramos, s/n. Cidade Universitária, Recife, PE. CEP 50670-901. E-mail: villareluna@yahoo.com.br

${ }^{3}$ Departamento de Ciências G eográficas/U FPE. Av. Acadêmico Hélio Ramos, s/n, Cidade U niversitária, CEP 50741-530, Recife, PE. Fone: (81) $2126-7376$. E-mail: bbdasilva.ufpe@gmail.com; josicleda@hotmail.com

${ }^{4}$ DECIC/U FPE. E-mail: suzanam@ufpe.br
} 


\section{INTRODUÇÃO}

No Brasil existe grande diversidade de biomas sendo o cerrado o segundo maior em relação à sua extensão territorial, perdendo apenas para a Floresta Tropical. Este bioma possui diferentes fisionomias, desde a formação campestre (campo limpo, campo cerrado) até florestas com dossel fechado em composição com sub-bosques (cerrado sensu stricto, cerradão). Apesar de ser um bioma de grande importância, o Cerrado está sob o arco do desmatamento, que cresce de forma desordenada e acelerada. O desmatamento ocasiona grandes consequências além dos impactos já esperados relacionados à perda da fauna e da flora, alterações climáticas significativas. Nobre et al. (1999) afirmam que o efeito do desmatamento em grande escala pode provocar alterações no clima, na hidrologia e nos ciclos biogeoquímicos em escalas regional e global. Silva et al. (2011) relatam que alterações no uso da terra na Caatinga, com a introdução de cultivos irrigados, alteraram os fluxos radiativos com consequente aumento da cobertura vegetal e acentuada redução na temperatura da superfície.

$\mathrm{O}$ elemento mais importante a influenciar processos atmosféricos e as condições do tempo e clima no planeta Terra, é a energia proveniente do Sol, responsável pelo aquecimento do ar e do solo, fotossíntese e evaporação da água. As variações no saldo de radiação $(\mathrm{Rn})$ são fundamentais nos processos atmosféricos e terrestres alterando, por exemplo, a temperatura na superfície, o perfil da taxa vertical de aquecimento e a circulação atmosférica (Souza et al., 2008). Neste sentido, são de suma importância os estudos relacionados ao saldo de radiação; além do mais, ele é necessário no planejamento de atividades agrícolas irrigadas e na estimativa das perdas de água em superfícies vegetadas. De acordo com Silva et al. (2005b), Rn exerce papel fundamental nos processos de troca de calor e massa na baixa troposfera, uma vez que constitui o principal responsável pelo aquecimento do solo, do ar e, sobremaneira, pela evapotranspiração da vegetação nativa e das culturas.

$\mathrm{O} \mathrm{Rn}$ resulta no balanço entre os fluxos radiativos descendentes e ascendentes de onda curta e onda longa, que atuam na superfície e depende bastante da radiação solar global, do albedo, temperatura e emissividade da superfície, apresentando variação temporal e espacial que impactam fortemente as trocas de calor e massa na baixa atmosfera. Sua determinação direta pode ser feita com saldo radiômetros; no entanto, essas medições são representativas de áreas de pequena dimensão e boa homogeneidade e, ademais, os custos de aquisição e manutenção são elevados, o que restringe sua ampla utilização (Silva et al., 2011). Portanto, a determinação do Rn em escala regional e áreas heterogêneas tem sido possível com o emprego de imagens de sensores orbitais. Visando suprir esta grande lacuna, têm sido formulados diferentes algoritmos destinados à obtenção do Rn e demais componentes do balanço de energia à superfície com imagens orbitais, alguns dos quais requerem grande número de informações para serem empregados adequadamente. Bisht et al. (2005) propuseram um modelo de balanço de radiação diário senoidal baseado em uso de produtos extraídos de imagens do sensor MODIS MODerate resolution Imaging Spectroradiometer, do satélite
Terra. Uma séria limitação do algoritmo é que o mesmo se aplica apenas aos dias sem a presença de nuvens em todo o dia sendo, portanto, de aplicação prática muito limitada. Considerandose que a estimativa do $\mathrm{Rn}$ diário com o referido modelo depende do Rn instantâneo, sua extrapolação na obtenção do Rn diário sem a utilização de qualquer elemento do tempo diário, pode ocasionar erro considerável. Tang \& Li (2008) desenvolveram um método de regressão estatístico destinado ao cômputo do saldo de radiação de onda longa instantâneo, baseado em radiância registrada pelo MODIS no topo da atmosfera terrestre. Long et al. (2010) combinaram produtos MODIS, imagem de modelo de elevação digital e dados meteorológicos de superfície na modelagem dos balanços de onda curta e onda longa, em região ao norte do território chinês. Muitos estudos têm sido dedicados à determinação do Rn instantâneo e/ou diário, através do sensoriamento remoto. Podem ser ainda destacados os trabalhos de Wang \& Liang (2009), Lu \& Zhuang (2010), Jin et al. (2011) e Santos (2011), que avaliaram obalanço por meio de imagens MODIS. Já Lagouarde et al. (1991) estimaram Rn através de imagens AVHRR, enquanto Gratton et al. (1993), Silva et al. (2005b), Allen et al. (2007), Hong et al. (2009), Gomes et al. (2009) e Silva et al. (2011) utilizaram imagens TM-Landsat 5 para estimativas do Rn na superfície. Referidos estudos evidenciam a importância do monitoramento ambiental, em particular das trocas radiativas entre superfície e atmosfera, por meio de imagens orbitais. Neste sentido, o presente trabalho tem por objetivo avaliar o desempenho de balanço de radiação instantâneo unicamente com imagens TM-Landsat 5 e a obtenção do saldo diário com imagens TM, complementadas apenas com radiação solar global diária medida em superfície, em área de grande importância ecológica centralizada pela Ilha do Bananal, TO.

\section{Material E MÉTODOS}

A área de estudo compreende a Ilha do Bananal, localizada no Brasil Central, no estado do Tocantins, e que faz fronteira com os estados de Goiás, Mato Grosso e Pará. Como a maior ilha fluvial do mundo (com cerca de dois milhões de hectares), esta ilha é uma faixa de transição entre a Floresta Amazônica e o Cerrado (Rezende et al., 2001). Trata-se de uma área de várzea (alagável sazonalmente), classificada como ecótono: uma zona de tensão ecológica entre comunidades ou biomas adjacentes que sofrem mútua interferência e onde ocorre grande diversidade genética (Oliveira, 2006). O clima predominante nesta ilha é tropical quente semiúmido, com temperatura máxima de $38{ }^{\circ} \mathrm{C}$ no período das secas (meses de agosto a setembro) e temperatura mínima de $22^{\circ} \mathrm{C}$ no mês de julho. O período das cheias do rio Araguaia inunda parte da Ilha do Bananal e áreas adjacentes, durante os meses de janeiro a maio.

As imagens TM-Landsat 5 selecionadas para a pesquisa foram obtidas junto ao INPE - Instituto Nacional de Pesquisas Espaciais, correspondendo à órbita 223 e ponto 67, dos seguintes dias do ano de 2005: 3 de junho, 21 de julho, 6 de agosto e 22 de agosto. O recorte selecionado possui as seguintes coordenadas: canto superior esquerdo de $9^{\circ} 24^{\prime}$ 26,53 " S e $50^{\circ} 02^{\prime} 11,54$ " W e canto inferior direito de $9^{\circ} 59^{\prime}$ ' $51^{\prime \prime}$ 
Tabela 1. Variáveis utilizadas no cômputo do saldo de radiação e suas componentes: hora (tempo central GMT), ângulo de elevação do sol (E), o inverso do quadrado da distância relativa Terra-Sol (dr), cosseno do ângulo zenital solar (Z), radiação solar global diária (Rs, 24 h), temperatura do ar (Ta), radiação solar global instantânea (Rs)

\begin{tabular}{|c|c|c|c|c|c|c|c|c|}
\hline Data & DOA & Hora (GMT) & $E\left({ }^{\circ}\right)$ & $\mathrm{Dr}$ & $\operatorname{Cos} Z$ & $R s, 24 h^{\left(W ~ m^{-2}\right)}$ & $\mathrm{Ta}\left({ }^{\circ} \mathrm{C}\right)$ & $\operatorname{Rs}\left(W m^{-2}\right)$ \\
\hline $03 / 06 / 2005$ & 154 & $13: 12: 56$ & 45,90 & 0,97 & 0,718 & 225,0 & 28,2 & 710,7 \\
\hline $21 / 07 / 2005$ & 202 & $13: 13: 19$ & 45,73 & 0,97 & 0,716 & 229,3 & 30,3 & 714,6 \\
\hline 06/08/2005 & 218 & $13: 13: 26$ & 48,21 & 0,97 & 0,746 & 248,3 & 29,8 & 762,6 \\
\hline $22 / 08 / 2005$ & 234 & $13: 13: 34$ & 51,67 & 0,98 & 0,784 & 242,9 & 31,1 & 780,5 \\
\hline
\end{tabular}

S e 49 33' 54,96" W, ilustrado na Figura 1. Para validação dos resultados foram utilizadas medições de saldo radiômetro (Kipp-Zonen) instalado em torre micrometeorológica montada na Ilha do Bananal, a $2 \mathrm{~km}$ a leste do rio Araguaia, em local com as seguintes coordenadas: $9^{\circ} 49^{\prime} 16^{\prime \prime} \mathrm{S}, 50^{\circ} 08^{\prime} 55^{\prime \prime} \mathrm{W}$ e $120 \mathrm{~m}$ de altitude (Oliveira, 2006). As variáveis utilizadas para o cômputo do saldo e suas componentes estão representadas na Tabela 1.

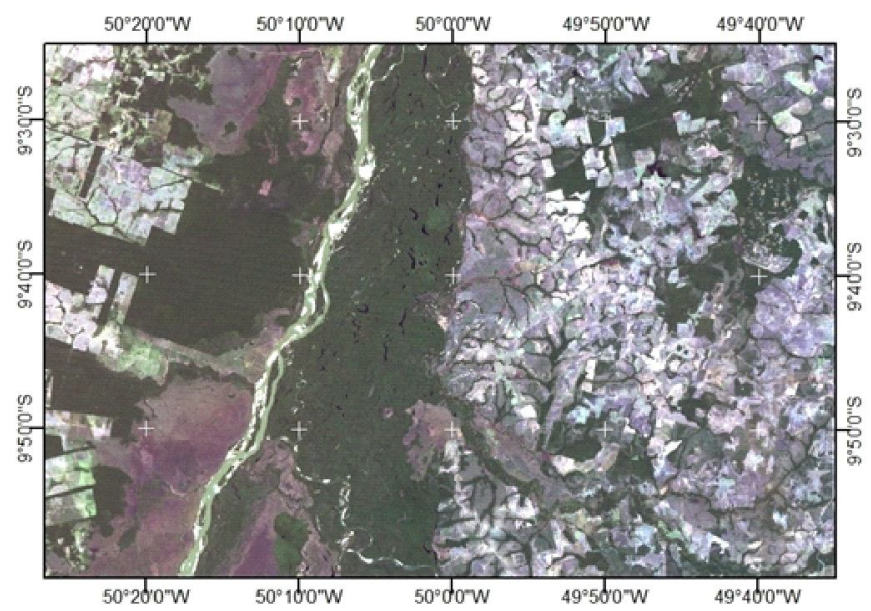

Figura 1. Imagem do dia 03/06/2005 em composição RGB123 do TM -Landsat5, com destaque para a localização da torre micrometeorológica na llha do Bananal e com os respectivos recortes para as áreas $\mathrm{A} 1$ e A2

A obtenção do saldo de radiação com imagens TM-Landsat 5 compreende uma sequência de etapas, descritas a seguir.

\section{Calibração radiométrica}

Compreende o processo de transformação do número digital (ND) de cada pixel em radiância espectral $\left(\mathrm{L}_{\lambda_{\mathrm{i}}}\right)$, que representa a energia solar refletida por cada pixel por unidade de área, tempo, ângulo sólido e de comprimento de onda, medida o nível do satélite, sendo obtido por (Chander et al., 2009):

$$
\mathrm{L}_{\lambda \mathrm{i}}=\mathrm{a}_{\mathrm{i}}+\frac{\mathrm{b}_{\mathrm{i}}-\mathrm{a}_{\mathrm{i}}}{255} \mathrm{ND}
$$

em que:

$\mathrm{L}_{\lambda \mathrm{i}}$ - radiância espectral de cada banda

a e b - radiâncias mínima e máxima obtidas mediante processo de calibração do TM - Landsat 5 cujos valores estão mostrados na Tabela 2

$\mathrm{ND}$ - número digital (número inteiro entre 0 a 255) e i corresponde às bandas $(1,2,3, \ldots$ e 7$)$ do TM-Landsat 5
Tabela 2. Constantes de calibração e Irradiância solar espectral no topo da atmosfera para o TM-Landsat 5 (Chander et al., 2009)

\begin{tabular}{|c|c|c|c|}
\hline \multirow{2}{*}{ Banda } & a & b & \multirow{2}{*}{$K_{\lambda \mathrm{i}}\left(W m^{-2} \mu m^{-1}\right)$} \\
\hline & \multicolumn{2}{|c|}{$\left(W m^{-2} s r^{-1} \mu m^{-1}\right)$} & \\
\hline 1 & $-1,52$ & 193,0 & 1957,0 \\
\hline 2 & $-2,84$ & 365,0 & 1826,0 \\
\hline 3 & $-1,17$ & 264,0 & 1554,0 \\
\hline 4 & $-1,51$ & 221,0 & 1036,0 \\
\hline 5 & $-0,37$ & 30,2 & 215,0 \\
\hline 6 & 1,2378 & 15,303 & - \\
\hline 7 & $-0,15$ & 16,5 & 80,67 \\
\hline
\end{tabular}

\section{Reflectância monocromática}

A refletância monocromática $\left(\rho_{\lambda_{\mathrm{i}}}\right)$ no topo da atmosfera para cada uma das bandas do visível e infravermelho, é dada pela razão entre o fluxo de radiação solar refletido e o fluxo de radiação solar incidente (Chander \& Markham, 2003), dado por:

$$
\rho_{\lambda \mathrm{i}}=\frac{\pi \cdot L_{\lambda i}}{K_{\lambda i} \cdot \cos Z \cdot d r}
$$

em que:

$\mathrm{L}_{\lambda \mathrm{i}}$ - radiância monocromática de cada banda do $\mathrm{TM}, \mathrm{W} \mathrm{m}^{-2}$ $\mathrm{sr}^{-1} \mu \mathrm{m}^{-1}$

$\mathrm{K}_{\lambda_{\mathrm{i}}}$ - irradiância solar monocromática de cada banda, $\mathrm{W} \mathrm{m}^{-2}$ $\mu \mathrm{m}^{-1}$, no topo da atmosfera (Tabela 2)

$\mathrm{Z}$ - ângulo zenital do sol

dr - inverso do quadrado da distância relativa terra-sol

\section{Albedo planetário}

O albedo planetário $\left(\alpha_{\text {plan }}\right)$ representa o albedo de cada pixel sem correção atmosférica, que consiste em combinação linear da reflectância espectral de cada uma das seis bandas reflectivas e seus respectivos pesos $\left(\mathrm{w}_{\mathrm{b}}\right)$ :

$$
\begin{aligned}
\alpha_{\mathrm{plan}} & =\mathrm{w}_{\lambda 1} \cdot \rho_{\lambda 1}+\mathrm{w}_{\lambda 2} \cdot \rho_{\lambda 2}+\mathrm{w}_{\lambda 3} \cdot \rho_{\lambda 3}+ \\
& +\mathrm{w}_{\lambda 4} \cdot \rho_{\lambda 4}+\mathrm{w}_{\lambda 5} \cdot \rho_{\lambda 5}+\mathrm{w}_{\lambda 7} \cdot \rho_{\lambda 7}
\end{aligned}
$$

em que:

$\mathrm{w}_{\lambda}$ - dado pela razão entre $\mathrm{o} \mathrm{K}_{\lambda \mathrm{i}}$ de cada banda e o somatório de todos os valores de $\mathrm{K}_{\lambda \mathrm{i}}$

\section{Albedo da superfície}

Representa o albedo com correção dos efeitos da atmosfera, obtido segundo Bastiaanssen et al. (1998), qual seja:

$$
\alpha_{\text {sup }}=\left(\frac{\alpha_{\text {plan }}-\mathrm{a}}{\tau_{\mathrm{sw}}^{2}}\right)
$$


em que:

a - reflectância atmosférica, que no presente estudo foi considerada 0,03 (Bastiaanssen, 2000; Silva et al., 2005a, b; Silva et al., 2011);

$\tau_{\mathrm{SW}}$ - transmissividade atmosférica para condição de céu claro, obtida em função da altitude de cada pixel, segundo Allen et al. (2007).

\section{Índices de vegetação}

São utilizados para avaliar impactos decorrentes da ocupação humana nas áreas de estudo e na estimativa das emissividades. Obteve-se o Índice de Vegetação Ajustado aos Efeitos do Solo (SAVI) segundo Huete (1988):

$$
\text { SAVI }=\frac{(1+L)\left(\rho_{4}-\rho_{3}\right)}{\left(L+\rho_{4}+\rho_{3}\right)}
$$

em que:

L - constante de ajuste ao solo cujo valor usado no estudo foi 0,1 (Allen et al., 2007; Silva et al., 2011)

Na sequência, obteve-se o Índice de Área Foliar (IAF), definido pela razão entre a área foliar de toda a vegetação por unidade de área utilizada por essa vegetação, constituindo um indicador da biomassa de cada pixel, que foi computado pela equação (Allen et al., 2007):

$$
\mathrm{IAF}=-\frac{\ln \left(\frac{0,69-\mathrm{SAVI}}{0,59}\right)}{0,91}
$$

De acordo com a expressão anterior o valor máximo atingido pelo IAF é igual a 6,0, ocorrendo quando o SAVI vale 0,69 .

\section{Emissividades}

Os pixels não emitem radiação eletromagnética como um corpo negro razão pela qual, ao se calcular a temperatura da superfície, necessita-se calcular sua emissividade $\left(\varepsilon_{\mathrm{NB}}\right)$ no domínio espectral da banda termal do TM - Landsat 5 (10,4 $12,5 \mu \mathrm{m})$. Para tanto, fez-se uso da expressão parametrizada por Allen et al. (2007):

$$
\varepsilon_{\mathrm{NB}}=0,97+0,0033 \times \mathrm{IAF}
$$

Apesar disto, para calcular a radiação de onda longa emitida por cada pixel deve-se considerar a emissividade $\varepsilon_{0}$ em todo o domínio da onda longa (4 a $100 \mu \mathrm{m})$, qual seja:

$$
\varepsilon_{0}=0,95+0,01 \times \mathrm{IAF}
$$

Essas equações são válidas para SAVI $>0$ e IAF $\leq 3$. Para pixels com IAF $\geq 3, \varepsilon_{\mathrm{NB}}=\varepsilon_{0}=0,98$ e para corpos de água (SAVI $<0$ ) consideram-se $\varepsilon_{\mathrm{NB}}=0,99$ e $\varepsilon_{0}=0,985$ (Allen et al., 2007).

\section{Temperatura da superfície}

A temperatura da superfície - Ts é obtida com base na radiância espectral da banda $6\left(\mathrm{~L}_{\lambda 6}\right)$ e emissividade da banda termal $\left(\varepsilon_{\mathrm{NB}}\right)$, por meio da Lei de Planck invertida, segundo Markham \& Barker (1986):

$$
\mathrm{T}_{\mathrm{S}}=\frac{\mathrm{K}_{2}}{\ln \left(\frac{\varepsilon_{\mathrm{NB}} \mathrm{K}_{1}}{\mathrm{~L}_{\lambda, 6}}+1\right)}
$$

em que:

$\mathrm{K}_{1}$ e $\mathrm{K}_{2}$ - constantes de radiação específicas para o TMLandsat 5 , respectivamente iguais a $607,76 \mathrm{~W} \mathrm{~m}^{-2} \mathrm{sr}^{-1} \mu \mathrm{m}^{-1} \mathrm{e}$ $1260,56 \mathrm{~K}$.

Radiação de onda longa emitida por cada pixel e pela atmosfera A radiação de onda longa emitida por cada pixel $-\mathrm{R}_{\mathrm{L}}\left(\mathrm{W} \mathrm{m} \mathrm{m}^{-2}\right)$ foi calculada segundo a equação de Stefan-Boltzmann:

$$
\mathrm{R}_{\mathrm{L} \uparrow}=\varepsilon_{0} \sigma \mathrm{T}_{\mathrm{S}}^{4}
$$

em que $\varepsilon_{0}$ é a emissividade de cada pixel, ó é a constante de Stefan-Boltzmann $\left(\sigma=5,67 \times 10^{-8} \mathrm{~W} \mathrm{~m}^{-2} \mathrm{~K}^{-4}\right)$ e Ts é a temperatura da superfície $(\mathrm{K})$; já a radiação de onda longa emitida pela atmosfera na direção da superfície $\left(\mathrm{R}_{\mathrm{L}}\right)$, também foi obtida pela equação anterior, com a Ts sendo substituída pela média aritmética de Ts de toda a área de estudo e a emissividade atmosférica $-\varepsilon_{\mathrm{a}}$ obtida segundo Bastiaanssen et al. (1998), qual seja:

$$
\varepsilon_{\mathrm{a}}=\mathrm{a}\left(-\ln \tau_{\mathrm{SW}}\right)^{\mathrm{b}}
$$

em que:

a e b - coeficientes de calibração cujos valores, de acordo com Allen et al. (2007) são iguais, respectivamente, a 0,85 e 0,09

$\tau_{\mathrm{sw}}$ - transmitância atmosférica à radiação de onda curta

\section{Radiação de onda curta incidente}

A radiação de onda curta incidente (Rs) que atinge a superfície terrestre em condição de céu claro, para imagens com área espacial menor que $25.000 \mathrm{~km}^{2}$, pode ser obtida, segundo Allen et al. (2007), por:

$$
\mathrm{R}_{\mathrm{S \downarrow}}=\mathrm{S} \cdot \cos \mathrm{Z} \cdot \mathrm{dr} \cdot \tau_{\mathrm{sw}}
$$

em que:

S - constante solar, $1367 \mathrm{~W} \mathrm{~m}^{-2}$

Z - ângulo zenital solar

dr - inverso do quadrado da distância relativa terra-sol

$\tau_{\mathrm{SW}}$ - transmissividade atmosférica

\section{Saldo de radiação instantâneo}

O saldo de radiação instantâneo à superfície $\mathrm{Rn}\left(\mathrm{W} \mathrm{m}^{-2}\right)$ foi obtido por meio da seguinte equação:

$$
\mathrm{Rn}=(1-\alpha) \mathrm{R}_{\mathrm{S} \downarrow}+\mathrm{R}_{\mathrm{L} \downarrow}-\mathrm{R}_{\mathrm{L} \uparrow}-\left(1-\varepsilon_{0}\right) \mathrm{R}_{\mathrm{L} \downarrow}
$$

em que:

$\mathrm{R}_{\mathrm{S}}$ - radiação de onda curta incidente, $\mathrm{W} \mathrm{m}^{-2}$

$\alpha$ - albedo da superfície, adimensional 
$\mathrm{R}_{\mathrm{L}}$ - radiação de onda longa da atmosfera, $\mathrm{W} \mathrm{m} \mathrm{m}^{-2}$

$\mathrm{R}_{\mathrm{L}}$ - radiação de onda longa emitida por cada pixel, $\mathrm{W} \mathrm{m}^{-2}$

$\varepsilon_{0} \quad$ - emissividade do pixel

\section{Saldo de radiação diário}

Obteve-se o saldo de radiação diário - $\mathrm{Rn}_{24 \mathrm{~h}}\left(\mathrm{~W} \mathrm{~m} \mathrm{~m}^{-2}\right)$ por meio de modelo proposto por Bruin (1987), qual seja:

$$
\mathrm{Rn}_{24 \mathrm{~h}}=(1-\alpha) \mathrm{R}_{\mathrm{S} \downarrow 24 \mathrm{~h}}-110 \tau_{\mathrm{sw} 24 \mathrm{~h}}
$$

em que:

$$
\alpha \text { - albedo }
$$

$\mathrm{R}_{\mathrm{S} 24 \mathrm{~h}}$ - radiação solar global diária, $\mathrm{W} \mathrm{m}^{-2}$

$\tau_{\mathrm{sw} 24 \mathrm{~h}}$ - transmissividade atmosférica média diária obtida pela razão entre a radiação solar global diária $-\mathrm{R}_{\mathrm{S} 24 \mathrm{~h}}, \mathrm{MJ} \mathrm{m}^{-2}$

radiação solar diária incidente no topo da atmosfera $\mathrm{R}_{\text {STOA24h }}, \mathrm{MJ} \mathrm{m}^{-2}$

\section{Parâmetros estatísticos utilizados}

Para a análise dos resultados foram utilizados o Erro Médio Absoluto (EMA), o Erro Médio Percentual (EMP) e a Raiz do Erro Quadrático Médio (REQM):

$$
\begin{gathered}
\mathrm{EMA}=\frac{1}{\mathrm{~N}} \sum_{\mathrm{i}=1}^{\mathrm{N}}\left|\mathrm{X}_{\mathrm{i}}^{\prime}-\mathrm{X}_{\mathrm{i}}\right| \\
\mathrm{EMP}=\frac{100}{\mathrm{~N}} \sum_{\mathrm{i}=1}^{\mathrm{N}}\left|\frac{\mathrm{X}_{\mathrm{i}}^{\prime}-\mathrm{X}_{\mathrm{i}}}{\mathrm{X}_{\mathrm{i}}}\right| \\
\operatorname{REQM}=\left(\frac{\sum_{\mathrm{i}}^{\mathrm{N}}\left(\mathrm{X}^{\prime}-\mathrm{X}\right)^{2}}{\mathrm{~N}}\right)^{1 / 2}
\end{gathered}
$$

em que:

$\mathrm{X}_{\mathrm{i}}(\mathrm{Rn}, \mathrm{obs})$ - corresponde ao valor do $\mathrm{Rn}$ medido

$X^{\prime}(\mathrm{Rn}$, sebal) - corresponde ao valor do $\mathrm{Rn}$ estimado segundo o SEBAL

$\mathrm{N}$ - corresponde ao número de pares de dados de $\mathrm{Rn}$

\section{RESULTADOS E DISCUSSÃO}

Os valores médios do saldo de radiação instantâneo - Rn,inst ( $\mathrm{W} \mathrm{m}^{-2}$ ) obtidos sobre todo o recorte estudado, variaram de 535,4 a 489,5 $\mathrm{W} \mathrm{m}^{-2}$, que correspondem aos dias 22/08/2005 e $21 / 07 / 2005$, respectivamente. Os valores mais elevados do Rn,inst foram localizados nos corpos d'água, que apresentaram valores acima de $575 \mathrm{~W} \mathrm{~m}^{-2}$. Bastiaanssen et al. (1998) registraram valores próximos a $500 \mathrm{~W} \mathrm{~m}^{-2} \mathrm{em}$ oásis. No Lago de Sobradinho, estado da Bahia, Silva et al. (2005b) obtiveram $751,3 \mathrm{~W} \mathrm{~m}^{-2}$. No estado de São Paulo Gomes et al. (2009) encontraram, para espelhos d'água, valores superiores a 731 $\mathrm{W} \mathrm{m} \mathrm{m}^{-2}$. Os valores encontrados pelos autores foram consideravelmente altos em comparação com os do presente estudo, provavel-mente pela diferença entre as localizações das áreas de pesquisa e pelas diferentes propriedades físicas e bioquímicas da água nessas áreas. Deve-se registrar, ainda, que o $\mathrm{Rn}$ em corpos d'água depende de vários fatores, tais como: ângulo de incidência dos raios solares, profundidade e turbidez da água, dentre outros.

$\mathrm{Na}$ vegetação nativa foram encontrados valores que se diferiram um pouco entre os dias estudados. Na imagem do dia 03/06/2005 foram obtidos valores do Rn,inst entre 525 e 550 $\mathrm{W} \mathrm{m^{-2 }}$ e, para o dia 21/07/200,5 os valores diminuíram para 500 e $525 \mathrm{~W} \mathrm{~m}^{-2}$. Na imagem do dia 06/08/2005 o Rn,inst ficou entre 550 e $575 \mathrm{~W} \mathrm{~m}^{-2}$ e na imagem do dia 22/08/2005 foram encontrados valores maiores que $575 \mathrm{~W} \mathrm{~m}^{-2}$ e outros pontos com valores entre 550 e $575 \mathrm{~W} \mathrm{~m}^{-2}$. Gomes et al. (2009) registraram, para o cerrado (no estado de São Paulo) valores entre 628,5 e 376,2 $\mathrm{W} \mathrm{m}^{-2}$. Esses valores corroboram com os resultados obtidos no presente estudo apresentando apenas um aumento nos valores máximos o que se deve, provavelmente, à maior incidência dos raios solares.

Em áreas antropizadas os valores encontrados do Rn estão situados, de modo geral, entre 425 a $500 \mathrm{~W} \mathrm{~m}^{-2}$, com alguns pontos que variaram entre $375 \mathrm{a} 425 \mathrm{~W} \mathrm{~m}^{-2}$. Os bancos de areia no leito do rio Araguaia se apresentaram para todas as imagens com valor inferior a $375 \mathrm{~W} \mathrm{~m}^{-2}$. Bastiaanssen et al. (1998) obtiveram, para o deserto $\mathrm{Rn}$, em torno de $400 \mathrm{~W} \mathrm{~m}^{-2}$. Silva et al. (2005b) obtiveram, para Juazeiro, BA, e Petrolina, PE, em solo exposto, valor de 421,8 $\mathrm{W} \mathrm{m}^{-2}$ enquanto naquela mesma área Di Pace et al. (2008) obtiveram, para solo exposto nos anos de 2000 e 2001, valores de 431,4 e 312,6 W m², respectivamente. Gomes et al. (2009) registraram, em áreas que sofrem ação antrópica em Santa Rita do Passa Quatro, SP, valores entre 300 e $400 \mathrm{~W} \mathrm{~m}^{-2}$, resultados menores aos aqui apresentados. Já Chavez et al. (2007) obtiveram, para solos descobertos, valores entre 500 e $550 \mathrm{~W} \mathrm{~m}^{-2}$. Ressalta-se que esses valores dependem de outros fatores, como estoque de umidade do solo, sazonalidade da radiação solar e interferência das chuvas locais.

Analisando os resultados das diferentes áreas pode-se verificar que na área da vegetação nativa foi encontrado maior valor do saldo instantâneo, se comparado com as áreas antropizadas indicando uma energia disponível maior para ser repartida nos processos de aquecimento do ar (calor sensível), do solo (calor no solo) e fluxo de massa (calor latente).

Comparando visualmente as quatro imagens estudadas (Figura 2) diferenciam-se claramente áreas de corpos d'água, vegetação nativa e áreas antropizadas e, de acordo com as cartas apresentadas, percebe-se que houve uma visível diferença entre os dias estudados, resultado da forte influência da radiação solar global instantânea (Tabela 1).

Na Tabela 3 se encontram os valores instantâneos do saldo de radiação obtidos pelo SEBAL e os medidos na torre micrometeorológica. Verifica-se variação do saldo de radiação instantâneo entre 525,4 a 586,8 W m² e que houve uma variação significativa do saldo para os dias estudados.

Destaca-se que o saldo radiômetro possui um erro instrumental de $2,5 \%$ e, dependendo da altura em que esteja instalado em relação à superfície de estudo, tem cobertura equivalente a um círculo com raio que varia de 5 a $15 \mathrm{~m}$, o que 
A.

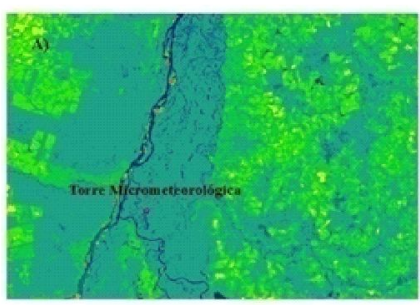

C.
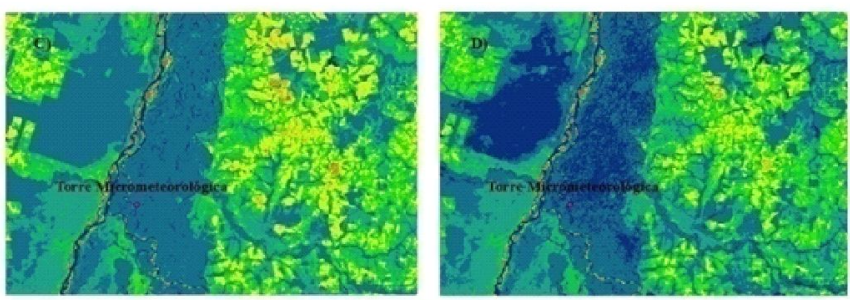

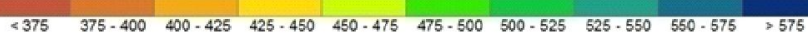

Figura 2. Saldo de radiação instantâneo obtido através do sensor TM para os seguintes dias de estudo: 03/06/2005 (A), 21/07/2005 (B), 06/08/2005 (C) e 22/08/2005 (D)

Tabela 3. Valores medidos e estimados do Saldo de Radiação Instantâneo - Rn,ins (W $\mathrm{m}^{-2}$ ) para os dias de estudo e seus respectivos erros relativo (ER) e absoluto (EA)

\begin{tabular}{cccccc}
\hline Data & DOA & Rn,inst,med & Rn,inst,SEBAL & $\begin{array}{c}\text { ER } \\
\text { (\%) }\end{array}$ & $\begin{array}{c}\text { EA } \\
\left(\text { W } \mathbf{~ m}^{-2} \text { ) }\right.\end{array}$ \\
$03 / 06 / 2005$ & 154 & 559,9 & 560,3 & 0,07 & 0,39 \\
$21 / 07 / 2005$ & 202 & 551,6 & 525,4 & 4,75 & 26,22 \\
$06 / 08 / 2005$ & 218 & 587,2 & 563,3 & 4,06 & 23,86 \\
$22 / 08 / 2005$ & 234 & 583,7 & 586,8 & 0,53 & 3,07 \\
\hline \multicolumn{7}{c}{ Média } & & 2,35 & 13,38 \\
\hline
\end{tabular}

seria equivalente a um pixel do TM. Em vários estudos com imagens TM, o Erro Relativo Médio é inferior a 5\% e, considerando-se uma imagem TM completa, com cerca de 38 milhões de pixels, constata-se a grande importância da obtenção do Rn com imagens TM. Neste estudo foram obtidos erros relativos que variaram entre 0,1 a $4,8 \%$. Gomes et al. (2009) encontraram erros relativos que variaram entre 0,4 a $14,3 \%$, bem superiores aos obtidos nesta pesquisa, indicando que a metodologia aqui apresentada se mostra bastante eficaz. $\mathrm{O}$ erro relativo médio aqui registrado foi de $2,4 \%$, com erro absoluto médio de $13,4 \mathrm{~W} \mathrm{~m}^{-2} \mathrm{e}$ REQM de apenas $17,8 \mathrm{~W} \mathrm{~m}^{-2}$. Bisht et al. (2005) obtiveram, com imagens MODIS REQM $=74 \mathrm{~W} \mathrm{~m}^{-2}$, enquanto Ryu et al. (2008) registraram valores de $65 \mathrm{~W} \mathrm{~m}^{-2}$, Wang \& Liang (2009) de 17,72 W m². Já em Bisht \& Bras (2010) foram reportados valores do REQM de $37 \mathrm{~W} \mathrm{~m}^{-2}$ enquanto em Jin et al. (2011) de $30 \mathrm{~W} \mathrm{~m}^{-2}$, nestes últimos estudos foram também utilizadas imagens MODIS. Os dados obtidos pelos autores mencionados foram superiores aos aqui encontrados, exceto os de Wang \& Liang (2009), que encontraram valores ligeiramente inferiores, confirmando a eficácia da metodologia proposta no presente estudo.

Já o saldo de radiação diário - $\mathrm{Rn}_{, 24 \mathrm{~h}}$ obtido nesta pesquisa, apresentou valores médios entre 132,7 e 145,2 $\mathrm{W} \mathrm{m}^{-2}$ que corresponderam, respectivamente, aos dias 21/07/2005 e 06/ 08/2005. Na Figura 3 observam-se as cartas geradas para o
A.

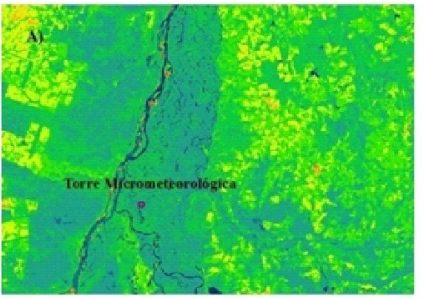

C.
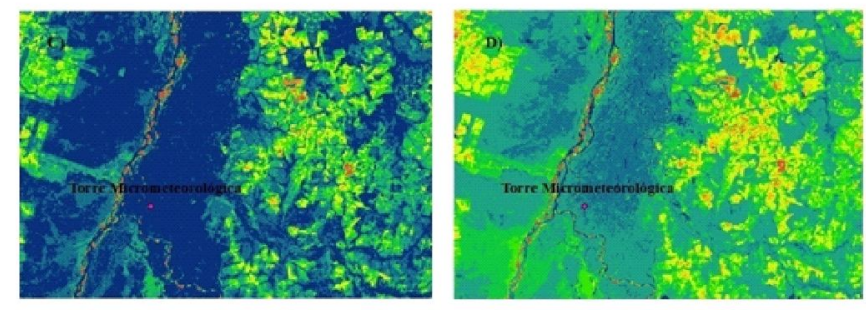

$=110 \quad 110 \cdot 115 \quad 115 \cdot 120 \quad 120 \cdot 125 \quad 125=130 \quad 130 \cdot 135 \quad 135 \cdot 140 \quad 140 \cdot 145 \quad 145 \cdot 150 \quad>150$ Figura 3. Representação do saldo de radiação diário obtido através de imagens do sensor TM para os seguintes dias de estudo: 03/06/2005 (A), 21/07/2005 (B), 06/08/ 2005 (C) e 22/08/2005 (D)

$\mathrm{Rn}_{.24 \mathrm{~h}}$ para todos os dias estudados evidenciando que os padrões do $\mathrm{Rn}_{24 \mathrm{~h}}$ são absolutamente idênticos aos observados nas cartas do Rn,inst.

Os dados do saldo diário medidos na torre corroboram com os valores apresentados nas cartas geradas do saldo de radiação diário. Os maiores valores do $\mathrm{Rn}_{24 \mathrm{~h}}$ foram verificados em corpos d'água superiores a $150 \mathrm{~W} \mathrm{~m}^{-2}$, seguidos dos valores obtidos de superfícies cobertas por vegetações nativas (cerrado) que se apresentou, para cada dia de estudo, de forma particular. Na imagem do dia 03/06/2005 as áreas de vegetação registraram $\mathrm{Rn}_{.24 \mathrm{~h}}$ entre 130 a $140 \mathrm{~W} \mathrm{~m}^{-2}$, a do dia 21/07/2005 entre 130 e $135 \mathrm{~W} \mathrm{~m}^{-2}$, aquela de $06 / 08 / 2005 \mathrm{Rn}_{24 \mathrm{~h}}$, maiores que $150 \mathrm{~W} \mathrm{~m}^{-2}$ em sua grande maioria; por fim, no dia 22/08/2005 se obtiveram valores de $\mathrm{Rn}_{24 \mathrm{~h}}$ entre 140 e $150 \mathrm{~W} \mathrm{~m}^{-2}$. Em geral, os menores valores de $\mathrm{Rn}$ diário ocorreram nas áreas antropizadas que apresentaram $\mathrm{Rn}$ entre 120 e $135 \mathrm{~W} \mathrm{~m}^{-2}$ (de forma geral) apresentando, ainda, pequenos focos com valores menores que $110 \mathrm{~W} \mathrm{~m}^{-2}$. Silva et al. (2011) encontraram valores de $\mathrm{Rn}_{.24 \mathrm{~h}}$ entre 173,4 a 196,6 W m² e 95,6 a 112,5 W m² para o Açude São Gonçalo e solo exposto no estado da Paraíba, respectivamente, em ocasião de maior oferta de radiação solar.

As cartas geradas através do sensor TM mostram clara diferenciação entre as áreas supracitadas (rio, áreas antropizadas e vegetação nativa). No canto superior esquerdo de cada imagem ficou bem evidente a diminuição do saldo de radiação diário à superfície, percebendo-se claramente a ocupação antrópica, com seus limites bem definidos em todas as imagens analisadas. Observa-se, no lado direito da imagem, a ocupação humana, de forma que dá para visualizar a diminuição do $\mathrm{Rn}_{, 24 \mathrm{~h}}$, mas não fica estabelecido claramente um padrão, ao longo das imagens. Pode-se avaliar, através das cartas apresentadas, que a Ilha do Bananal está sob o arco do desmatamento, o que influencia diretamente o clima local, haja vista que um saldo maior de radiação está diretamente associado a um albedo pequeno, maior quantidade de água e/ 
ou maior índice de vegetação e menor Ts, que reúnem condições para proporcionar alto valor do fluxo de calor latente e baixo calor sensível. Em contrapartida, um albedo aumentado com a presença humana (material em suspensão na água, estresse hídrico da planta e a sua própria retirada), em geral reduz o Rn e os índices de vegetação e contribui para aumentar a Ts e o fluxo de calor sensível, resultando em aumento na temperatura do ar.

Na Tabela 4 se apresentam os valores medidos e estimados do saldo de radiação diário com seus respectivos erros relativos e absolutos. Pode-se verificar que esses resultados obtidos foram bastante significativos de vez que os erros apresentados foram muito pequenos.

Tabela 4. Valores medidos e estimados do saldo para os dias de estudo e seus respectivos erros relativo (ER) e absoluto (EA)

\begin{tabular}{cccccc}
\hline Data & DOA & Rn,24h_med & Rn,24h_SEBAL & $\begin{array}{c}\text { ER } \\
\text { (\%) }\end{array}$ & $\begin{array}{c}\text { EA } \\
\left(\mathbf{W} \mathbf{~ m}^{-2} \text { ) }\right.\end{array}$ \\
$03 / 06 / 2005$ & 154 & 138,6 & 139,5 & 0,65 & 0,90 \\
$21 / 07 / 2005$ & 202 & 138,4 & 137,5 & 0,65 & 0,90 \\
$06 / 08 / 2005$ & 218 & 146,5 & 151,5 & 3,41 & 5,00 \\
$22 / 08 / 2005$ & 234 & 139,5 & 143,8 & 3,08 & 4,30 \\
\hline \multicolumn{7}{c}{ Média } & & 1,95 & 2,78 \\
\hline
\end{tabular}

Os erros relativo e absoluto médios foram de 1,95\% e 2,78 $\mathrm{W} \mathrm{m}{ }^{-2}$, respectivamente, enquanto a Raiz do Erro Quadrático Médio (REQM) foi de 3,36 $\mathrm{W} \mathrm{m}^{-2}$. Bisht et al. (2005) obtiveram, em sua pesquisa com $\mathrm{Rn}_{.24 \mathrm{~h}}$ REQM de $50 \mathrm{~W} \mathrm{~m}^{-2}$ e Bisht \& Bras (2010) registraram REQM igual a $38 \mathrm{~W} \mathrm{~m}^{-2}$, em estudos com imagens MODIS. Esses resultados da REQM dos autores mencionados foram bem superiores ao encontrado no presente estudo devido, muito provavelmente, ao pressuposto de $\mathrm{Rn}$ nulo em todo o período noturno que, conceitualmente, não estaria adequado.

\section{CONCLUSÕES}

1. O método proposto para estimar o saldo de radiação instantâneo à superfície, mostrou-se bastante eficaz, não carecendo de parâmetros de entrada obtidos em superfície e foi capaz de estimar o saldo de radiação instantâneo à superfície com pequenos erros relativos e absolutos; por outro lado, o saldo de radiação diário com uso de imagens de satélite e apenas a radiação solar global diária, resultaram em erros menores que os observados em Rn,inst, demonstrando a consistência e a importância da técnica empregada.

2. Foi possível avaliar, espacialmente, as mudanças ocorridas no saldo de radiação e, em consequência, os impactos antrópicos sobre a temperatura do ar em áreas que não possuem informações micrometeorológicas.

\section{Agradecimentos}

Os autores agradecem ao Conselho Nacional de Desenvolvimento Científico e Tecnológico $(\mathrm{CNPq})$, pelo apoio financeiro a esta pesquisa e por bolsa de Produtividade e Pesquisa concedida aos segundo e terceiro autores e também à CAPES e ao CNPq, pela concessão de bolsa de pósgraduação ao primeiro autor.

\section{LITERATURA CITADA}

Allen, R. G.; Tasumi, M.; Trezza, R. Satellite-based energy balance for mapping evapotranspiration with internalized calibration (METRIC) - Model. Journal of Irrigation and Drainage Engineering, v.133, p.380-394, 2007.

Bastiaanssen, W. G. M. SEBAL-based sensible and latent heat fluxes in the irrigated Gediz Basin, Turkey. Journal of Hydrology, v.229, p.87-100, 2000.

Bastiaanssen, W. G. M.; Menenti, M.; Feddes, R. A.; Holtslag, A. A. M. A remote sensing surface energy balance algorithm for land (SEBAL). 1: Formulation. Journal of Hydrology, v.212-213, p.198-212, 1998.

Bisht, G.; Bras, R. L. Estimation of net radiation from the MODIS data under all sky conditions: Southern Great Plains case study. Remote Sensing of Environment, v.114, p.1522-1534, 2010.

Bisht, G.; Venturini, V.; Islam, S.; Jiang, L. Estimation of the net radiation using MODIS (Moderate Resolution Imaging Spectroradiometer) data for clear-sky days. Remote Sensing of Environment, v.97, p.52-67, 2005.

Bruin, H. A. R. de. From Penman to Makkink. In: Hooghart, J. C. (ed.), Proceedings and information: TNO Committee on Hydrological. Gravennhage, The Netherlands, v.39, p.5-31, 1987.

Chander, G.; Markham, B. Revised Landsat 5 - TM radiometric calibration procedures and post calibration dynamic ranges. IEEE Transactions on Geosciences and Remote Sensing, v.41, p.2674-2677, 2003.

Chander, G.; Markham, B. L.; Helder, D. L. Summary of current radiometric calibration coefficients for Landsat MSS, TM, ETM+, and EO-1 ALI sensors. Remote Sensing of Environment, v.113, p.893-903, 2009.

Chavez, J. L.; Gowda, P. H.; Marek, T. H.; New, L. L. Evapotranspiration mapping using METRIC ${ }^{\mathrm{TM}}$ for region with highly advective conditions. In: ASABE Annual International Meeting, 2007, Minneapolis. Anais... Minneapolis: ASABE, 2007. CD Rom.

Di Pace, F. T.; Silva, B. B. da; Silva, V. de P. R. da; Silva, S. T. A. da. Mapeamento do saldo de radiação com imagens Landsat 5 e modelo de elevação digital. Revista Brasileira de Engenharia Agrícola e Ambiental, v. 12, p.385-392, 2008.

Gomes, H. F., Silva, B. B. da, Cavalcanti, E. P., Rocha, H. R. Balanço de radiação em diferentes biomas no estado de São Paulo mediante imagens Landsat 5. Geociências, v.28, p.153$164,2009$.

Graton, D. J.; Howarth, P. J.; Marceau, D. J. Using Landsat 5 Thematic Mapper and digital elevation data to determine the net radiation field of a Mountain Glacier. Remote Sensing of Environment, v.43, p.315-331, 1993. 
Hong, S. H.; Hendrickx, J. M. H.; Borchers, B. Up-scaling of SEBAL derived evapotranspiration maps from Landsat (30 $\mathrm{m})$ to MODIS (250 m) scale. Journal of Hydrology, v.370, p.122-138, 2009.

Huete, A. R. A soil adjusted vegetation index (SAVI). Remote Sensing of Environment, v.25, p.295-309, 1988.

Jin, Y,; Randerson, J. T.; Goulden, M. L. Continental-scale net radiation and evapotranspiration estimated using MODIS satellite observations. Remote Sensing of Environment, v.115, p.2302-2319, 2011.

Lagouard, J. P.; Brunet, Y.; Kerr, Y.; Imbernon, J. Estimating the daily upward longwave radiation from NOAA-AVHRR data for mapping net radiation. Advances in Space Research, v.11, p.151-161, 1991.

Long, D.; Gao, Y.; Singh, V.P. Estimation of daily average net radiation from MODIS data and DEM over the Baiyangdian watershed in North China for clear sky days. Journal of Hydrology, v.388, p.217-233, 2010.

Lu, X. L.; Zhuang, Q. L. Evaluating evapotranspiration and wateruse efficiency of terrestrial ecosystems in the conterminous United States using MODIS and AmeriFlux data. Remote Sensing of Environment, v.114, p.1924-1939, 2010.

Markham, B. L.; Barker, J. L. Landsat MSS and TM postcalibration dynamic ranges, exoatmospheric reflectances and at satellite temperatures. EOSAT Landsat Technical Notes, Lanham: Earth Observation Satellite Company, v.1, p.3-8, 1986.

Nobre, C. A.; Fisch, G.; Nobre, A. D. Balanço de energia, vapor d'água e $\mathrm{CO} 2$ em áreas de floresta tropical na Amazônia Central. In: Relatório do projeto (0966/95) realizado pelo INPE e INPA, p.373-385, 1999.

Oliveira, L. S. Fluxos atmosféricos de superfície sobre uma área de ecótono na Ilha do Bananal. Tese Doutorado. São Paulo: USP, 2006. 114p.
Rezende, D.; Merlin, S.; Santos, M. Sequestro de carbono: uma experiência concreta. 2.ed. Aparecida de Goiânia: Poligráfica, 2001. 178p.

Ryu, Y.; Kang, S.; Moon, S. K.; Kim, J. Evaluation of land surface radiation balance derived from moderate resolution imaging spectroradiometer (MODIS) over complex terrain and heterogeneous landscape on clear sky days. Agricultural and Forest Meteorology, v.148, p.1538-1552, 2008.

Santos, F. A. C. Alterações no balanço radiativo e impactos climáticos decorrentes do uso da terra no estado de São Paulo. Campina Grande: UFCG, 83p. 2011. Dissertação Mestrado

Silva, B. B. da; Braga, A. C.; Braga, C. C. Balanço de radiação no perímetro irrigado São Gonçalo - PB mediante imagens orbitais. Revista Caatinga, v.24, p.145-152, 2011.

Silva, B. B. da; Lopes, G. M.; Azevedo, P. V. de. Determinação do albedo de áreas irrigadas com base em imagens Landsat 5 -TM. Revista Brasileira de Agrometeorologia, v.13, p.201211, 2005a.

Silva, B. B. da; Lopes, G. M.; Azevedo, P. V. Balanço de radiação em áreas irrigadas utilizando imagens Landsat 5-TM. Revista Brasileira de Meteorologia, v.20, p.243-252, 2005b.

Souza, J. D.; Silva, B. B. da; Ceballos, J. C. Estimativa da radiação solar global à superfície usando um modelo estocástico: caso sem nuvens. Revista Brasileira de Geofísica, v.26, p.3144, 2008.

Tang, B.; Li, Z. L. Estimation of instantaneous net surface longwave radiation from MODIS cloud-free data. Remote Sensing of Environment, v.112, p.3482-3492, 2008.

Wang, W.; Liang, S. Estimation of high-spatial resolution clearsky longwave downward and net radiation over land surfaces from MODIS data. Remote Sensing of Environment, v.113, p.745-754, 2009. 